\title{
An SHM approach using machine learning and statistical indicators extracted from raw dynamic measurements
}

\author{
Rafaelle Piazzaroli Finotti ${ }^{\text {a* }}$ \\ Alexandre Abrahão Cury ${ }^{b}$ \\ Flávio de Souza Barbosa $a^{a, b}$ \\ a Programa de Pós-Graduação em Modelagem Computacional, Faculdade de Engenharia, Universidade Federal de Juiz de Fora - Brazil. \\ E-mail: rafaelle.finotti@engenharia.ufjf.br, flavio.barbosa@ufjf.edu.br \\ ${ }^{\text {b }}$ Departamento de Mecânica Aplicada e Computacional, Faculdade de Engenharia, Universidade Federal de Juiz de Fora - Brazil. \\ E-mail:alexandre.cury@ufjf.edu.br \\ * Corresponding author
}

http://dx.doi.org/10.1590/1679-78254942

\begin{abstract}
Structural Health Monitoring using raw dynamic measurements is the subject of several studies aimed at identifying structural modifications or, more specifically, focused on damage assessment. Traditional damage detection methods associate structural modal deviations to damage. Nevertheless, the process used to determine modal characteristics can influence the results of such methods, which could lead to additional uncertainties. Thus, techniques combining machine learning and statistical analysis applied directly to raw measurements are being discussed in recent researches. The purpose of this paper is to investigate statistical indicators, little explored in damage identification methods, to characterize acceleration measurements directly in the time domain. Hence, the present work compares two machine learning algorithms to identify structural changes using statistics obtained from raw dynamic data. The algorithms are based on Artificial Neural Networks and Support Vector Machines. They are initially evaluated through numerical simulations using a simply supported beam model. Then, they are assessed through experimental tests performed on a laboratory beam structure and an actual railway bridge, in France. For all cases, different damage scenarios were considered. The obtained results encourage the development of computational tools using statistical indicators of acceleration measurements for structural alteration assessment.
\end{abstract}

\section{Keywords}

Structural Dynamic, Damage Identification, Computational Intelligence, Structural Health Monitoring, Vibration Monitoring, Dynamic Measurement.

\section{INTRODUCTION}

Structural alterations can be caused by many factors, such as design and constructive problems, operational conditions, severe natural events (earthquakes and storms), natural aging, etc. In most cases, changes in structures may be associated with damage. Structural Health Monitoring (SHM) allows detecting the presence of damage at an early stage, thus reducing maintenance costs and ensuring more comfort and safety to users. The interest in identifying structural damage has received considerable attention over the years and it is becoming increasingly important in engineering researches, mainly due to economic and security aspects. In this sense, different techniques based on vibration data to analyze structural changes have been explored, like those studied in Rytter (1993) and Doebling et al. (1998).

The main objective of SHM is to develop reliable and robust techniques focused on diagnosing a structural failure. Since degrading processes alter the structural dynamic behavior - by reducing its stiffness and modifying its vibrational 
characteristics - most damage identification and health monitoring methods are based on changes in modal parameters (i.e. natural frequencies, damping ratios and mode shapes). In this respect, several methods were developed: the Modal Assurance Criterion (MAC), employed as a correlation indicator between damaged and undamaged mode shapes (Allemang, 2003); the Strain Energy Method (SEM), an index based on mode shapes curvature with and without damage (Cury et al., 2011); analysis of flexibility matrix (Pandey \& Biswas, 1994), among others. A more comprehensive literature review of classical vibration-based techniques can be found in Doebling et al. (1998).

Despite their efficiency in numerical simulations, the aforementioned methods present some difficulties to assess the structural condition while dealing with experimental data in practical cases. Indeed, the identification process of modal parameters may lead to a loss of information or signal contamination, since it works as a filter, masking small structural alterations that can be related to damage. For instance, the spectral leakage phenomenon, characterized by spreading some frequency components over a bandwidth, is such a kind of signal distortion problem that occurs in the frequency domain during the modal identification process. Apart from that, modal parameters have the assumption that the structure only exhibits linear behavior, a consideration that is not applicable to all cases.

On the other hand, continuous monitoring programs have been increasingly used in civil engineering structures. The Rio-Niterói bridge in Brazil (Battista \& Pfeil, 2002), the Tsing-Ma bridge in China (Ni et al., 2011) and the Millau viaduct in France (Cachot et al., 2015), are just a few examples. Certainly, one of the most challenging aspects of identifying structural changes in a real-life monitoring system is to conduct a dynamic analysis during the normal operation of the structure, without controlling the input excitation. Ambient excitation sources, such as wind, traffic loads, or earthquakes, are uncontrollable and typically unknown. Therefore, structural inspection techniques based on actual vibration tests require signal processing algorithms capable of dealing with output-only measurements. Diverse sensing methods have been developed in this subject with the purpose of structural health monitoring, as in the pioneering work of Peeters \& De Roeck (1999) and, more recently, in the studies of Cardoso et al. (2017) and Cabboi et al. (2017). However, in the vast majority of these identification methods, dynamic data are often interpreted in function of their modal parameters, which can bring forth uncertainties to data, affecting the damage detection process, as previously discussed.

Thus, approaches interpreting raw dynamic responses using statistical analysis and computational intelligence for structural integrity assessment have been considered a promising field of research, as it can be seen in Alves et al. (2015), Amezquita-Sanchez \& Adeli (2016) and Santos et al. (2016). The main advantage of using raw dynamic responses is that the original data are directly processed, which circumvent effects caused by the modal parameter estimation process. Moreover, it is possible to deal with output-only measurements, since the knowledge of the vibration source is not necessary to assess structural behavior. Nevertheless, raw acceleration measurements are not generally used due to the volume of data and to the lack of tools capable of extracting relevant features from measured signals, usually noisy and complex. Thereby, the development of robust methods aimed at assessing the structural health condition by using raw dynamic data still demands a great effort.

Hence, the focus of this paper is to evaluate a vibration-based strategy to detect structural changes through statistical parameters extracted from raw acceleration measurements. Initially, ten different basic statistics are applied to characterize the dynamic data, by reducing $n$-dimensional time series (being $n$ the number of discrete time steps of the analysis) into 10-dimensional vectors, each one containing ten statistical data. Then, these statistical data vectors are used as inputs into two computational intelligence technologies: Artificial Neural Networks (ANN) and Support Vector Machines (SVM). The main idea is that the statistical indicators allow distinguishing apparently similar databases by inferring statistical properties about the data, whereas the computational methods can recognize similar observations and separate them into groups that share the same characteristics. The proposed methodology is analyzed and compared by using numerical simulation data of a simply supported beam and experimental data from tests performed on a beam, constructed in laboratory, and on an actual railway bridge in France.

Damage detection techniques focused on statistical indicators have been little explored in the literature. It is noteworthy that this work addresses a strategy that does not require any data filtering process. Also, since signal processing is also done in the time domain, distortion data problems due to modal identification do not influence on the present approach.

\section{STATISTICAL INDICATORS APPLIED TO VIBRATION DATA}

The determination of the input variables for the machine learning methods plays an essential role in the successful classification of different structural conditions. It is necessary to use data that provide representative information about the dynamic signals, since the classification methods learn through dataset mapping. 
According to Farrar \& Worden (2012), time series analysis of structural responses allows extracting signal parameters in a simpler way than modal analysis. With this in mind, this paper focuses on ten statistical parameters that permit working directly with dynamic measurements in the time domain (see Table 1).

Table 1: Statistical indicators.

\begin{tabular}{|c|c|}
\hline $\begin{array}{c}\text { Peak (SI1): } \\
x_{\text {peak }}=\max |\mathbf{x}|\end{array}$ & $\begin{array}{l}\text { Mean (S|2): } \\
\bar{x}=\frac{1}{n} \sum_{i=1}^{n} x_{i}\end{array}$ \\
\hline Mean square (SI3): & Root mean square (SI4): \\
\hline$x_{s q}=\frac{1}{n} \sum_{i=1}^{n}\left(x_{i}\right)^{2}$ & $\mathrm{rms}=\sqrt{\frac{1}{n} \sum_{i=1}^{n}\left(x_{i}\right)^{2}}$ \\
\hline Variance (SI5): & Standard deviation (SI6): \\
\hline$\sigma^{2}=\frac{1}{n} \sum_{i=1}^{n}\left(x_{i}-\bar{x}\right)^{2}$ & $\sigma=\sqrt{\frac{1}{n} \sum_{i=1}^{n}\left(x_{i}-\bar{x}\right)^{2}}$ \\
\hline Skewness (SI7): & Kurtosis (SI8): \\
\hline $\mathrm{s}=\underline{\frac{1}{n} \sum_{i=1}^{n}\left(x_{i}-\bar{x}\right)^{3}}$ & $\mathrm{k}=\underline{\frac{1}{n} \sum_{i=1}^{n}\left(x_{i}-\bar{x}\right)^{4}}$ \\
\hline$\sigma^{3}$ & $\mathrm{~K}=\overline{\sigma^{4}}$ \\
\hline Crest factor (SI9): & K-factor (SI10): \\
\hline $\mathrm{Cf}=\frac{x_{\text {peak }}}{\mathrm{rms}}$ & $\mathrm{Kf}=x_{\text {peak }} \cdot \mathrm{rms}$ \\
\hline
\end{tabular}

The first statistic is the peak amplitude that expresses the maximum oscillation magnitude of the signal. The mean, mean square and root mean square measure the central tendency of the data, while the standard deviation and variance measure their dispersion related to the mean value. Skewness and kurtosis are statistics of third and fourth order, respectively. The skewness represents the symmetry of data distribution and kurtosis indicates the flattening of the distribution considering a Gaussian curve. These statistics are mostly applied to random signals, except for the crest factor and the K-factor, frequently used to identify deviations from the sinusoidal response. Although some statistics are highly correlated, all of them are used as inputs to the classification algorithms, without employing any feature selection method beforehand.

\section{COMPUTATIONAL INTELLIGENCE METHODS APPLIED TO DAMAGE DETECTION}

Computational learning methods are considered useful tools for solving structural damage assessment problems. These algorithms work as classifiers that try to identify damage levels using as input data characteristics extracted from dynamic responses. Given all possible classification approaches, ANNs and SVMs are particularly well suited to address the issue of structural change detection, as it can be seen in Worden \& Lane (2001), Zang et al. (2004) and Alves et al. (2015). In the next sections some concepts about these computational methods are presented.

\subsection{Artificial Neural Network - ANN}

A popular computational intelligence technique for pattern recognition is the ANN, an adaptive learning machine trained to define the optimal decision boundaries among different classes of data, based on statistical mathematical models (Principe et al., 2000). The basic components of an ANN are the processing elements (PE), also called neurons. Due to its universal mapping capabilities, the Multilayer Perceptron (MLP) is the most commonly employed ANN architecture. The MLP consists in a feedforward ${ }^{1}$ topology composed of an input layer, one or more hidden layers and an output layer, which performs the input-output mapping through nonlinear functions. In summary, the neurons are interconnected and each connection has two associated adaptable parameters, weight and bias, adjusted by the backpropagation algorithm. The backpropagation transmits the error obtained in the output layer to the other layers and

\footnotetext{
${ }^{1}$ Unidirectional propagation of the signals among the neurons connections - from the input layer to the output layer.
} 
then updates the weights related to each MLP connection. The neurons take into account all connection contributions and produce an output that is a non-linear function of the sum of its inputs, aiming to minimize the error between the desired result and the network output. This iterative process is known as the training phase and stops when a specific criterion is achieved. After that, at the testing phase, the ANN model has to be able to generalize and recognize other inputs not seen during the training procedure.

It should be mentioned that the MLP is a universal approximator, in which one or two hidden layers are sufficient to describe almost any function. For pattern recognition problems, the use of only one hidden layer is usually enough. However, there are no specific or well-defined criteria in literature for choosing the best number of hidden layers (one or two) or the number of neurons. Typically, these parameters are empirically determined, selected by trial and error. More detailed information about ANN can be found in Principe et al. (2000).

\subsection{Support Vector Machine - SVM}

SVM is a statistical machine learning method that has been widely used to solve several pattern recognition problems. The fundamental idea of SVM is to construct an optimal separating hyperplane by maximizing the margin and minimizing the misclassification between two classes of data in a space (Vapnik, 1995). The SVM performs the classifications by defining some training data points positioned in the margins (support vectors), where the distance between them is maximized through an optimization function that minimizes the Euclidean norm of the hyperplane's directional vector. When dealing with nonlinear problems, the input variables are mapped in a higher-dimensional feature space by using a kernel function. The SVM theory can be extended to multi-class classification problems through strategies based on the combination of binary classifiers, e.g. one-against-one and one-against-all (Bishop, 2006).

In this work, the multi-class classification strategy "one-against-one" is adopted with the Radial Basis Function - RBF as the kernel. They are the most commonly used in the literature. In order to create an SVM model with RBF kernel, two parameters play an important role and have to be properly specified: $\sigma$ from the Gaussian kernel function and $\mathrm{C}$, a regularization parameter from the margin maximization formulation. These parameters are estimated by applying an exhaustive search procedure known as Grid Search. This procedure consists in training the SVM for different predetermined values of $\sigma$ and C. All $\sigma$ and C combinations are evaluated and the pair that presents the smallest inputoutput generalization error is selected. The $\sigma$ and $C$ values used in the Grid Search procedure of this study are given in Eq. (1) and Eq. (2), respectively.

$$
\begin{aligned}
& \sigma=\{ 0.0316,0.0398,0.0501,0.0631,0.0794,0.3162,0.3981 \\
& 0.5000,0.5012,0.6310,0.7943,1.0,1.5,2.0,2.5,3.0,3.5 \\
&4.0,4.5,5.0,5.5,6.0,6.5,7.0,7.5,8.0,8.5,9.0,9.5,10.0\} \\
& C=\{0.0316,0.3162,0.5000,1.0,1.5,2.0,2.5,3.0,3.5 \\
& 4.0,4.5,5.0,5.5,6.0,6.5,7.0,7.5,8.0,8.5,9.0 \\
&9.5,10.0,31.6228,100.0000,316.2278\}
\end{aligned}
$$

More detailed information about SVM can be found in Vapnik (1995) and Bishop (2006).

\section{STRUCTURAL BEHAVIOR ALTERATION DETECTION USING ACCELERATION RESPONSES}

Fig. 1 shows the proposed methodology, which can be described through the following steps:

1) The input data matrix is assembled with the statistical indicators, calculated using the entire set of acceleration time histories;

2) The ten statistical inputs and their labels (undamaged, damage - level 1, damage - level 2, etc...) are randomized (and also normalized if the ANN algorithm is used);

3) Some parameters are defined, such as the number of neurons, the training method, the activation and error function for the ANN, as well as the $\sigma$ and C values for the SVM (through Grid Search procedure);

4) The ANN and SVM are trained with a part of the dataset;

5) The rest of the data are used to test the model effectiveness, indicating different structural conditions. 


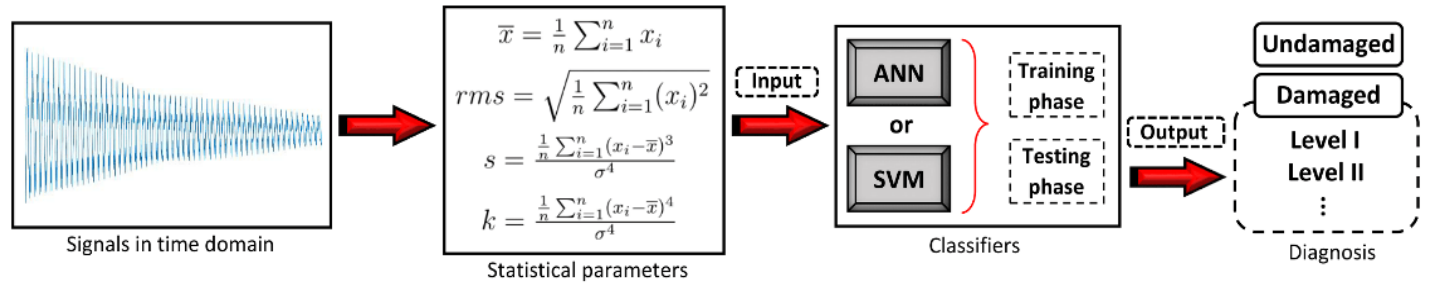

Figure 1: Scheme of the proposed methodology.

The present strategy is assessed through numerical and experimental tests. The computational intelligence algorithms, as well as the statistical indicators, were developed using toolboxes and built-in functions available in Matlab $\mathrm{R} 2016 \mathrm{~b}$. In order to reproduce the random numbers generated in the algorithms, the same seed was previously specified for all computational models of this work.

\subsection{Numerical test: simply supported beam model}

In this section, a numerical example using a finite element model (FEM) of a simply supported beam is chosen to verify the effectiveness of the proposed approach. The present FEM consists of 200 elements, where each element has two nodes with two degrees-of-freedom (vertical translation and rotation). The mechanical and geometrical properties are: the beam's length is $6 \mathrm{~m}$; Young's modulus (E) is equal to $210 \mathrm{GPa}$; the cross-section area is $2.81 \times 10^{-3} \mathrm{~m}^{2}$; the moment of inertia is equal to $1.845 \times 10^{-8} \mathrm{~m}^{4}$; and the mass density is $7850 \mathrm{~kg}$. $\mathrm{m}^{-3}$. A random force excitation $\mathrm{F}(\mathrm{t})$ with different amplitudes and frequencies was applied at $0.69 \mathrm{~m}$ from the right support. In an attempt to reproduce an actual instrumentation made using accelerometers, the vibration signals are simulated as vertical accelerations at ten equidistant measurement points (channels) collected during 100 s with a sampling frequency of $100 \mathrm{~Hz}$. The beam model is represented in Fig. 2.

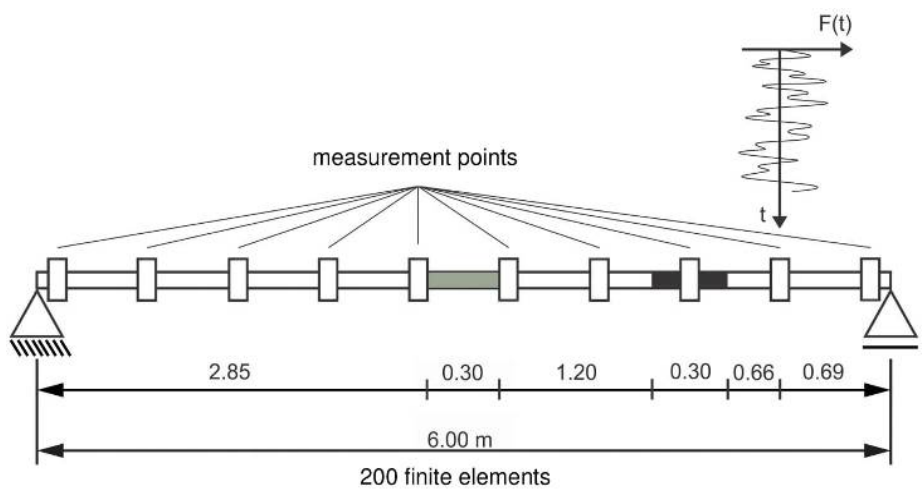

Figure 2: Numerical test of a simply supported beam (units: $m$ ).

A refinement study regarding the mesh division is shown in Fig. 3. The convergence of the relative error between the approximate and the exact theoretical natural frequencies is utilized to evaluate the finite element model. It is possible to observe that the error decreases significantly when the number of elements is greater or equal to 15 , approximately. As the required processing time for the analysis is not significant, 200 elements were used in the structural model discretization. This strategy permitted the evaluation of the proposed methodology by using different sets of simulated measured points, allowing practically identical results as the ones presented in this work. Since the optimization of the number and/or location of sensors is not focused on this work, this topic was not explored.

Three structural conditions are assumed: Healthy beam (undamaged); Damage - level 1 (characterized by a reduction of $20 \%$ in the modulus of elasticity at the midspan), illustrated by the gray region in Fig. 2; Damage - level 2 (characterized by a reduction of $10 \%$ in the modulus of elasticity at the quarter length), illustrated by the black region in Fig. 2, plus the second structural configuration (damage level 1). Moreover, the simulated time series are contaminated with the following random noise levels: no noise, $5 \%$ and $10 \%$, depicted by Eq. (3):

$x_{i, \text { noise }}=x_{i}+n_{\text {noise }} \cdot \sigma_{x_{i}} \cdot \mathrm{V} \sim \mathrm{N}(0,1)$ 
where $x_{i, \text { noise }}$ is the signal vector with noise, $x_{i}$ is the signal vector without noise, $n_{\text {noise }}$ is the random noise level, $\sigma_{x_{i}}$ is the standard deviation and $\mathrm{V} \sim \mathrm{N}(0,1)$ is a Gaussian vector with zero mean and unit standard deviation.

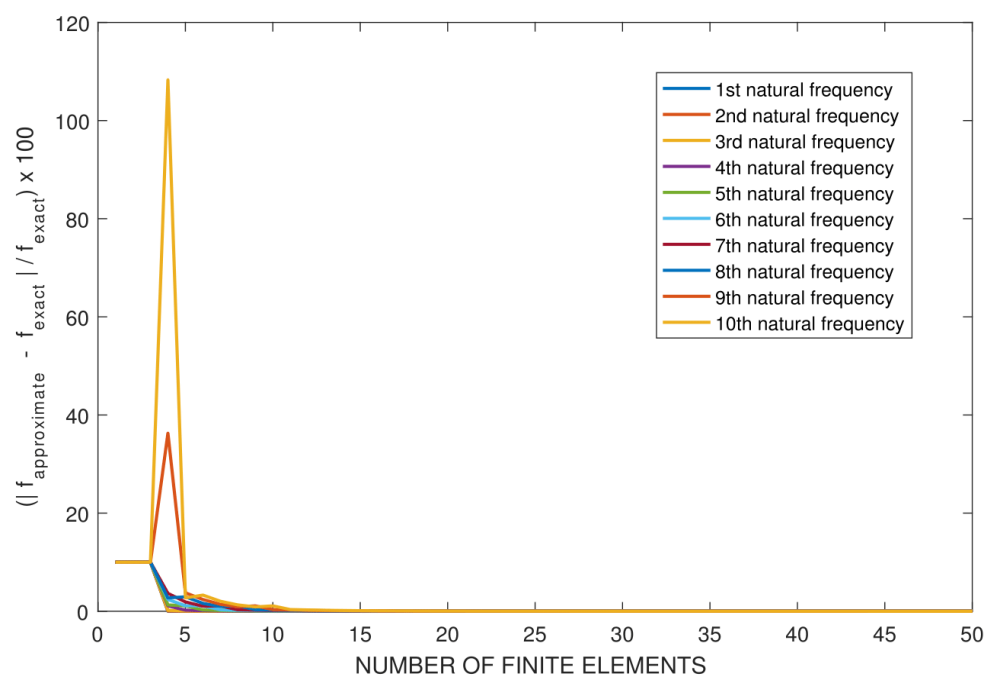

Figure 3: Refinement error convergence curve for the simply supported beam model.

In this study, ten different dynamic tests are simulated for each stage of damage and noise, resulting in 90 signals (10 vibration tests $\times 3$ structural conditions $\times 3$ noise levels). The excitation $F(t)$ (see Fig. 2) was randomly set for every single dynamic simulation. Fig. 4 shows an example of a typical response for the described simulation.

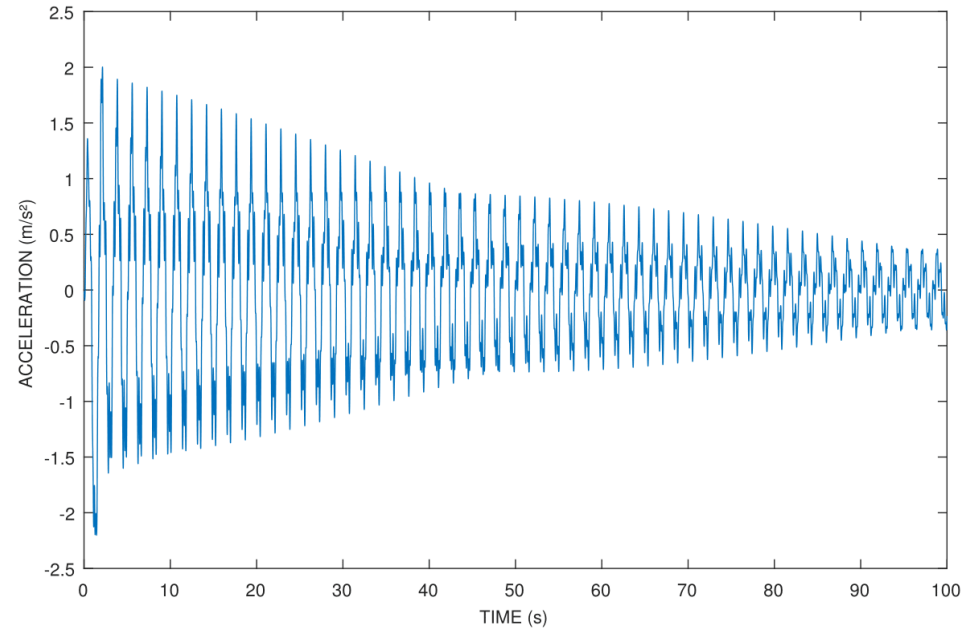

Figure 4: Typical response of the beam model.

All statistics are built using the entire set of acceleration time histories of each single simulated vibration test. Thus, the input dataset is organized in a matrix [90×100] as presented in Eq. (4), where the rows are related to the dynamic tests (data samples) and the columns to the statistical indicators (10 indicators $\times 10$ channels $=100$ features).

$$
\left[\begin{array}{cccc}
T_{1} S I_{1-m 1} & T_{1} S I_{1-m 2} & \cdots & T_{1} S I_{10-m 10} \\
T_{2} S I_{1-m 1} & T_{2} S I_{1-m 2} & \cdots & T_{2} S I_{10-m 10} \\
\vdots & \vdots & \ddots & \vdots \\
T_{90} S I_{1-m 1} & T_{90} S I_{1-m 2} & \cdots & T_{90} S I_{10-m 10}
\end{array}\right]
$$

Each set of ten columns has a type of statistical indicator, so the element $T_{1} S I_{1-m 1}$ corresponds to the statistical indicator $S I_{1}$ (peak) obtained at measurement point $m 1$ of the dynamic test $T_{1}$, the element $T_{1} S I_{1-m 2}$ corresponds to the 
statistical indicator $S I_{1}$ (peak) obtained at measurement point $m 2$ of the dynamic test $T_{1}$, and so on until the last element $T_{90} S I_{10-m 10}$, which corresponds to the indicator $S I_{10}$ (K-factor) at point $m 10$ of test $T_{90}$. In an effort to get a visual representation example of the input data, the variation of each statistical indicator over the three structural conditions at measurement point $m 5$ is shown in Fig. 5.

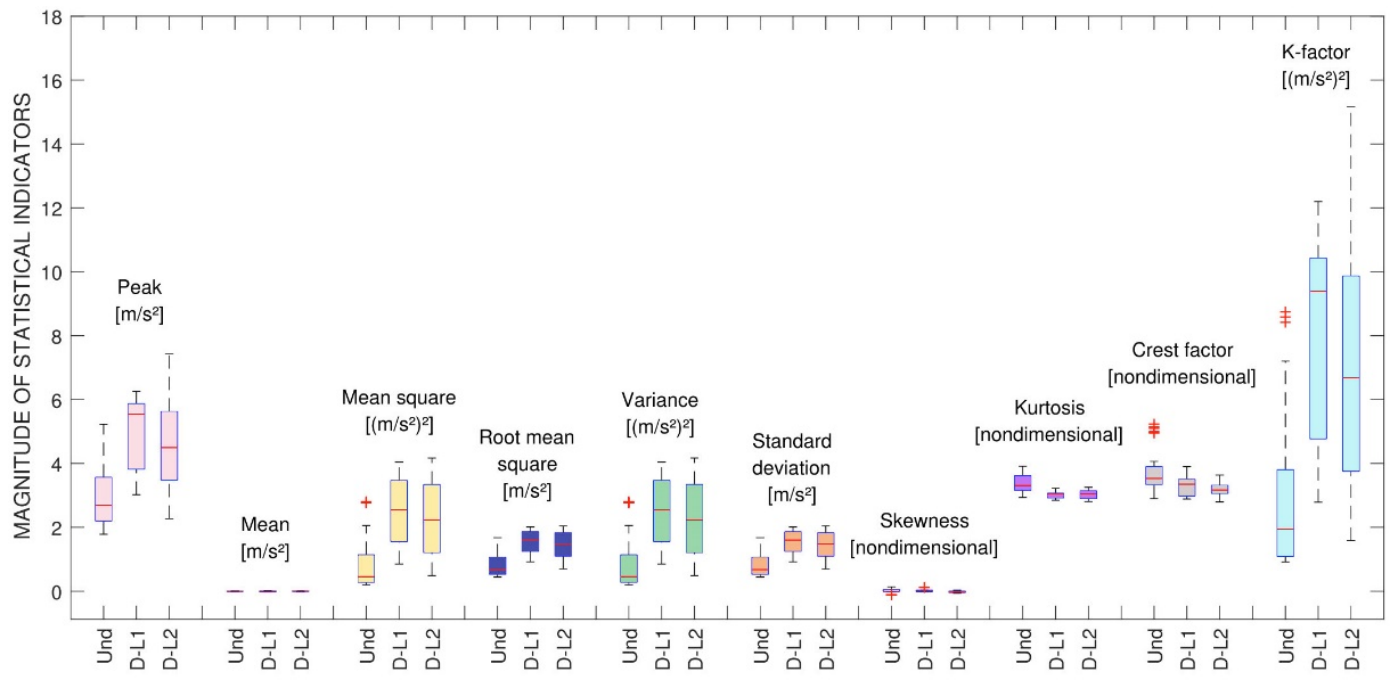

Figure 5: Variation of the ten statistical indicators for signals from measurement point $m 5$ of the beam model.

For the ANN method, the target data are arranged in a matrix [90×3], where the rows refer to the sample category

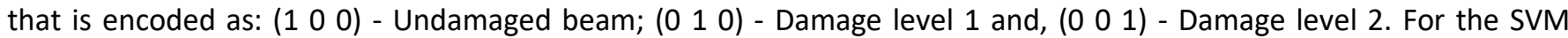
algorithm, the pattern representation of the input dataset is arranged in a target vector labeled as: 1 - Undamaged; 2 Damage level 1 and, 3 - Damage level 2.

The implemented neural network is an MLP with one hidden layer composed of 10 neurons, trained performing a 10-fold cross-validation (Kohavi, 1995), employing the Levenberg-Marquardt optimization method as training function (Hagan \& Menhaj, 1994), with an initial learning rate of $\mu=0.001$, using the logarithmic sigmoid as activation function and the mean square to estimate the error. The number of neurons was empirically selected, as discussed before (see in "Artificial Neural Network - ANN" subsection). Fig. 6 exhibits an illustration of the network architecture for the proposed ANN model.

It is important to mention that the 10 -fold cross-validation scheme aims to guarantee the generalization capacity of computational intelligence models when submitted to unseen data. The 10-fold is a consolidated validation technique that consists in randomly dividing the original dataset into 10 subsets - with approximately the same amount of data and examples of all cases - to create 10 different classification models in which distinct subsets are used for testing (1/10), validation (1/10) and training (8/10). Since each subset is used for testing only once, at the end of the ten iterations (folds), there is no model tested with overlapping data, ensuring reliable results. The overall performance is calculated by the accuracy average of the 10 models.

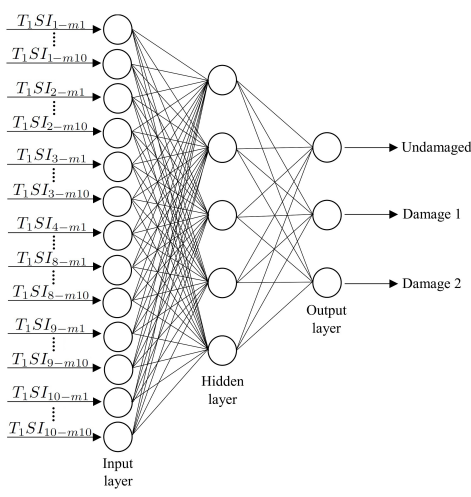

Figure 6: MLP network with 5 neurons in the hidden layer.

In order to check the developed neural network, a performance progress study is made on one of the classification models, considering the MSE error of dataset used for training, validation and testing stages, respectively. The 
performance of all generated classification models has the same trend, equivalent to the shown in Fig. 7. Generally, the training error becomes smaller as the training epochs increase. However, a large number of training epochs can also lead to a significant increase of the validation error, indicating a possible overfitted model that lost its capability to generalize unseen data. So, the best epoch refers to the iteration where the validation phase reached a minimum error value before its performance starts to decrease, working as network training stop criterion (after six consecutive increases in validation error). Since the training errors and validation/test error curves in Fig. 7 are very similar, no problem as underfitting or overfitting was found in this ANN classification model.

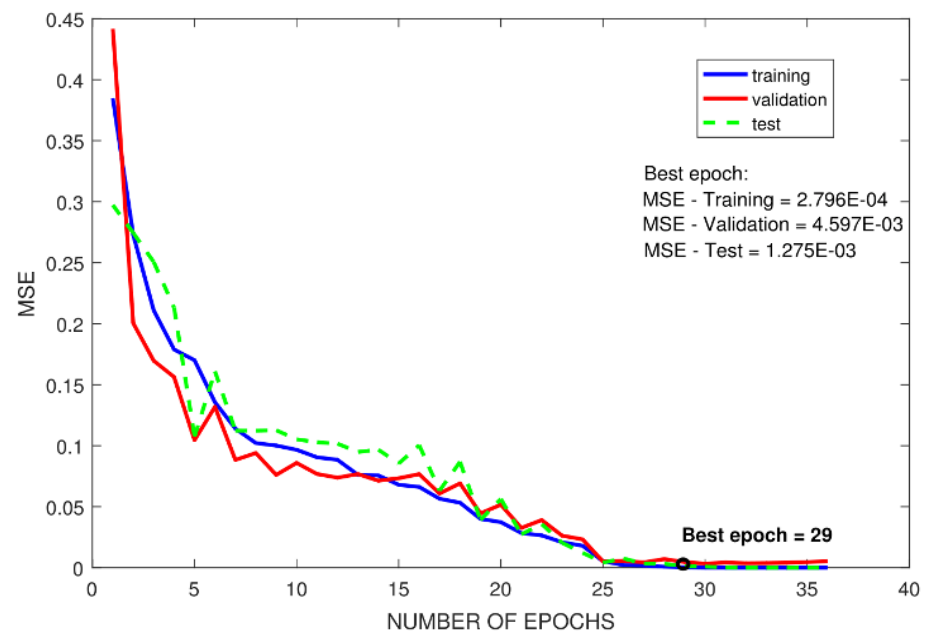

Figure 7: ANN performance progress for the beam model.

The SVM model was trained using as kernel the Radial Basis Function, where the best parameters $\sigma$ and $\mathrm{C}$ were chosen by evaluating multiple values in a 10 -fold cross-validation. Furthermore, the one-against-one strategy was employed to solve the multi-class classification problem (Bishop, 2006). For the SVM model of the present beam, the best parameters are $\sigma=3.00$ and $\mathrm{C}=8.5$. Differently from the ANN that uses empirical minimizations as a basis, the SVM works with structural risk minimization principle, which makes it less prone to overfitting. Since the SVM is an optimization technique that gives a unique and global solution, its performance evaluation is made only through the classification accuracy, presented in the following section.

\subsubsection{Results}

The performance of the ANN and SVM classifiers for the numerical beam model are presented in Table 2. The algorithms were executed 30 times in a 10-fold cross-validation. The classification rate or accuracy is the percentage of correct identifications divided by the number of executions and the final result is the mean of classification rates for the 30 iterations. Also, there is a classification confusion matrix, which presents the dispositions of the testing examples along with the different classes for the ANN and SVM algorithms. The "Output Class" corresponds to the predicted class and "Target Class" to the true class. The confusion matrices of the testing phases are represented in Fig. 8 and show the percentages of the models' overall accuracy, their precision (positive predictive value - the matrix's rightmost column) and their recall (true positive rate - the matrix's bottom row). These matrices were assembled taking into account all test subsets in the cross-validation scheme, resulting in 2700 different analyzed examples ( 9 examples for each test set $\times 10$ folds $\times 30$ models $=2700$ or 900 per structural condition).

The correct classification rates obtained by the ANN and SVM are greater than $80 \%$. Although both artificial intelligence methods were capable of detecting damage, the average classification performance of the SVM model is especially superior. Besides, the ANN standard deviation value is not the most suitable, almost reaching $5 \%$. For the same set of input data, the standard deviation decreased from $4.74 \%$ (ANN) to $1.04 \%$ (SVM) and the classification rate increased from $88 \%$ (ANN) to $99 \%$ (SVM), achieving 100\% sometimes (Table 2 - "max" value). A more comprehensive comparison among the results can be made by interpreting the confusion matrices in Fig. 8. The diagonal elements in green are the number and percentage of correct classifications for each class of the trained ANN and SVM. The accuracy percentage along the classes is slightly higher in SVM than in ANN, on average, 33\% against 30\%, respectively. For the ANN model, it is observed the worst performance is when 75 "undamaged signals" are incorrectly classified as "damaged signals - level 2", representing $2.8 \%$ of all tested dataset. Meanwhile, for the SVM model, the worst result is when 9 
"damaged signals - level 1" are classified as "damaged signals - level 2", corresponding to $0.3 \%$ of all tested dataset. These values confirm the better performance for the SVM classifier.

Table 2: Correct classification rate achieved for the simply supported numerical beam.

\begin{tabular}{ccccc}
\hline & Mean & $\begin{array}{c}\text { Standard } \\
\text { deviation }\end{array}$ & Max. & Min. \\
\hline ANN & $88.52 \%$ & $4.74 \%$ & $97.78 \%$ & $78.89 \%$ \\
SVM & $99.41 \%$ & $1.04 \%$ & $100.00 \%$ & $96.67 \%$ \\
\hline
\end{tabular}

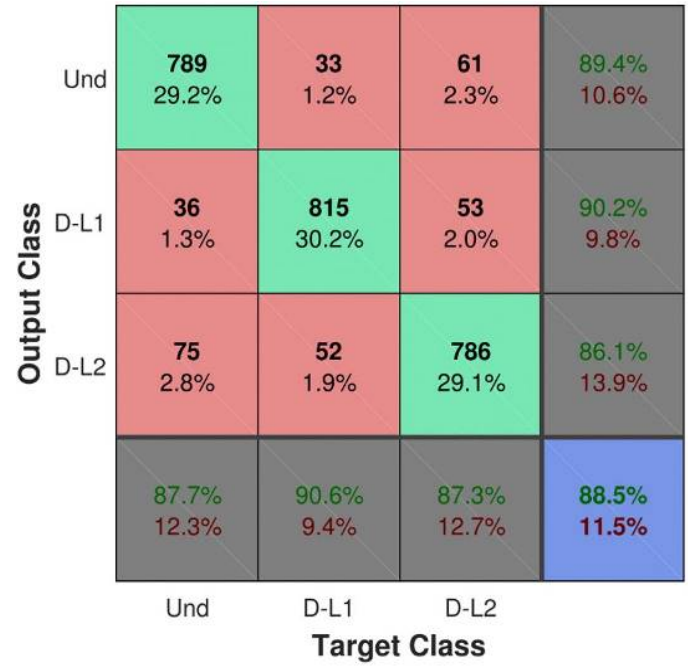

(a) ANN confusion matrix

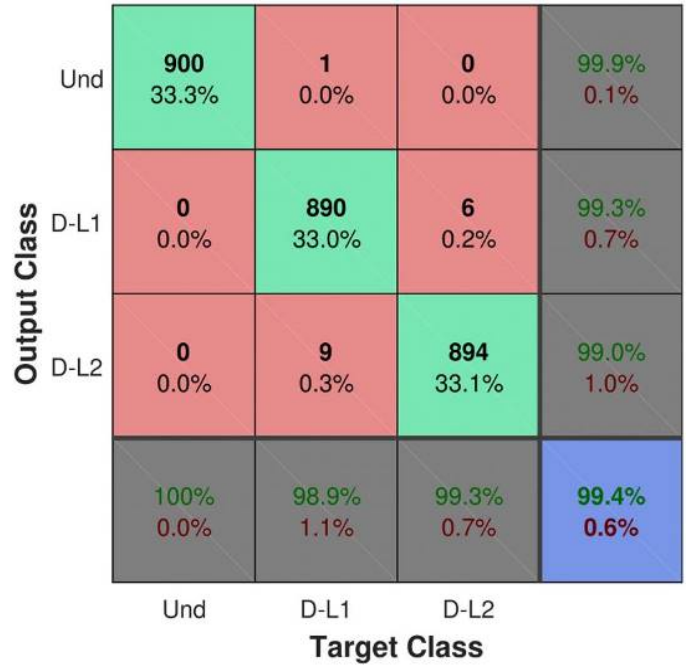

(b) SVM confusion matrix

Figure 8: Confusion matrices for the simply supported beam model.

\subsection{Experimental test I: steel beam}

The dynamic behavior of a simply supported steel beam is analyzed through an experimental investigation carried out in laboratory at Universidade Federal do Rio de Janeiro - COPPE (Alves et al., 2015). The studied beam has 1.46m long with a rectangular cross-section of $76.2 \mathrm{~mm} \times 8.0 \mathrm{~mm}$, as shown in Fig. 9 .

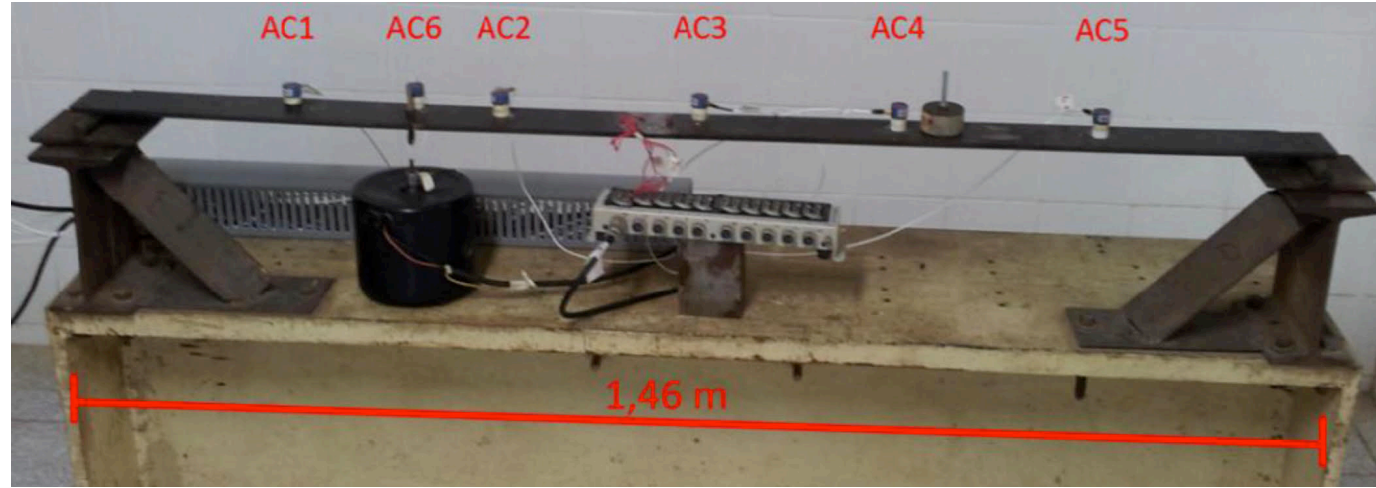

Figure 9: Experimental setup of the steel beam.

Dynamic tests were performed through random vibration excitations using a shaker. The dynamic signals were acquired by six piezoelectric accelerometers PCB model 336C31. Six acquisition campaigns were conducted trying to simulate very small structural damage. It is important to emphasize that these campaigns correspond to six different structural configurations (undamaged and damage levels 1, 2, 3, 4 and 5). The first scenario comprised the health beam (without damage). In order to reproduce a nonpermanent and reversible damage scenario, in the second campaign a $0.5 \mathrm{~kg}$ eccentric mass was positioned between the accelerometers AC5 and AC6, at $102.7 \mathrm{~cm}$ from the left support of the beam. For the third campaign, a $12-\mathrm{mm}$ round hole was imposed at the same location where the mass has been previously positioned but removed beforehand. The fourth, fifth and sixth campaigns consisted in increasing the hole to 
$16 \mathrm{~mm}, 22.5 \mathrm{~mm}$ and $32 \mathrm{~mm}$, respectively. In each campaign, three tests were recorded, resulting in 18 dynamic tests in total. Each test lasted around $10 \mathrm{~min}$ with at a sampling rate of $4000 \mathrm{~Hz}(0.00025 \mathrm{~s})$, respecting the Nyquist frequency. Fig. 10 shows a typical response of the experimental steel beam.

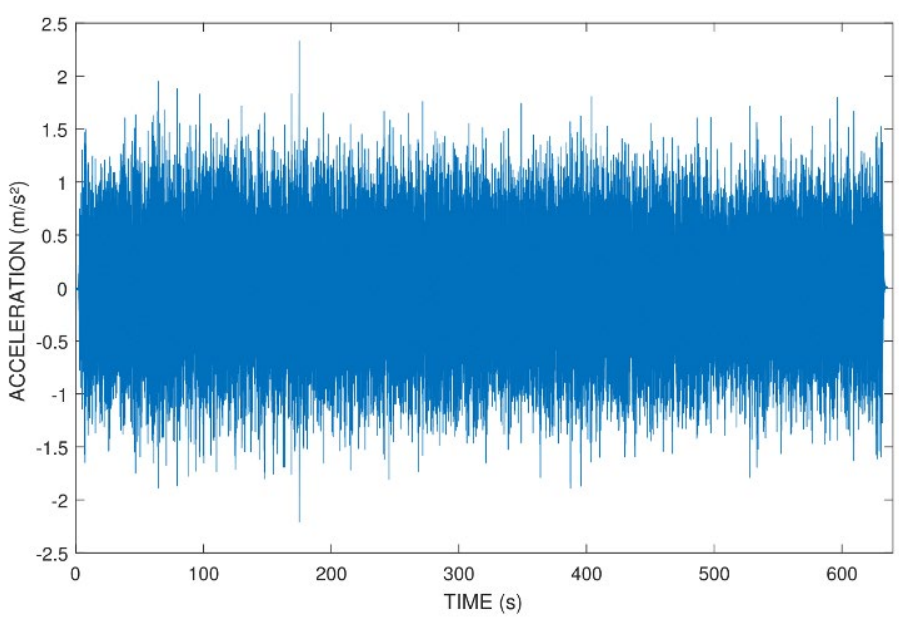

Figure 10: Typical response of the experimental beam.

At this point, it is worth remembering that the focus of this paper is to evaluate a strategy to identify structural changes in real-life monitoring during the normal operation of the structure, based on output-only measurements, without controlling the input excitations, as it happens in actual applications. Therefore, repeatability tests as well as the coherence analysis, usually performed when dealing with experimental dynamic data obtained under controlled ambient excitations, were not taken into account.

For better training of the damage detection models, the machine learning methods need a significant amount of data. For this reason, in the present work, the structural responses of $10 \mathrm{~min}$ were divided into 10 one-minute signals, providing an input matrix [ $180 \times 60]$ as represented in Eq. (5), where the rows correspond to the dynamic tests and the columns to the statistics ( 10 indicators $\times 6$ accelerometer channels $=60$ input parameters). The matrix elements of Eq. (5) have already been explained in the previous section. A variation analysis of the ten statistical parameters over the different structural damage levels is presented in Fig. 11. The output is represented by a [180×6] target matrix, encoded

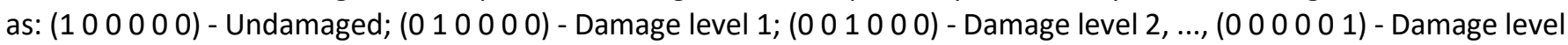
5.

The ANN model is an MLP with one-hidden layer which has 10 neurons trained by the Levenberg-Marquardt optimization method with an initial learning rate of $\mu=0.001$, using the 10 -fold cross-validation, the logarithmic sigmoid activation function and, the mean square to assess the error.

$$
\left[\begin{array}{cccc}
T_{1} S I_{1-m 1} & T_{1} S I_{1-m 2} & \cdots & T_{1} S I_{10-m 6} \\
T_{2} S I_{1-m 1} & T_{2} S I_{1-m 2} & \cdots & T_{2} S I_{10-m 6} \\
\vdots & \vdots & \ddots & \vdots \\
T_{180} S I_{1-m 1} & T_{180} S I_{1-m 2} & \cdots & T_{180} S I_{10-m 6}
\end{array}\right]
$$

The performance progress of the ANN classification models has the same trend, as shown in Fig. 12. In this case, there are no overfitting problems in the respective classification model. The validation curve approximately follows the training curve behavior until the epoch 12 (best epoch), in which the network stops the training stage preventing possible overfitting. From this epoch on, the validation error starts to increase consecutively as the training error decreases. 


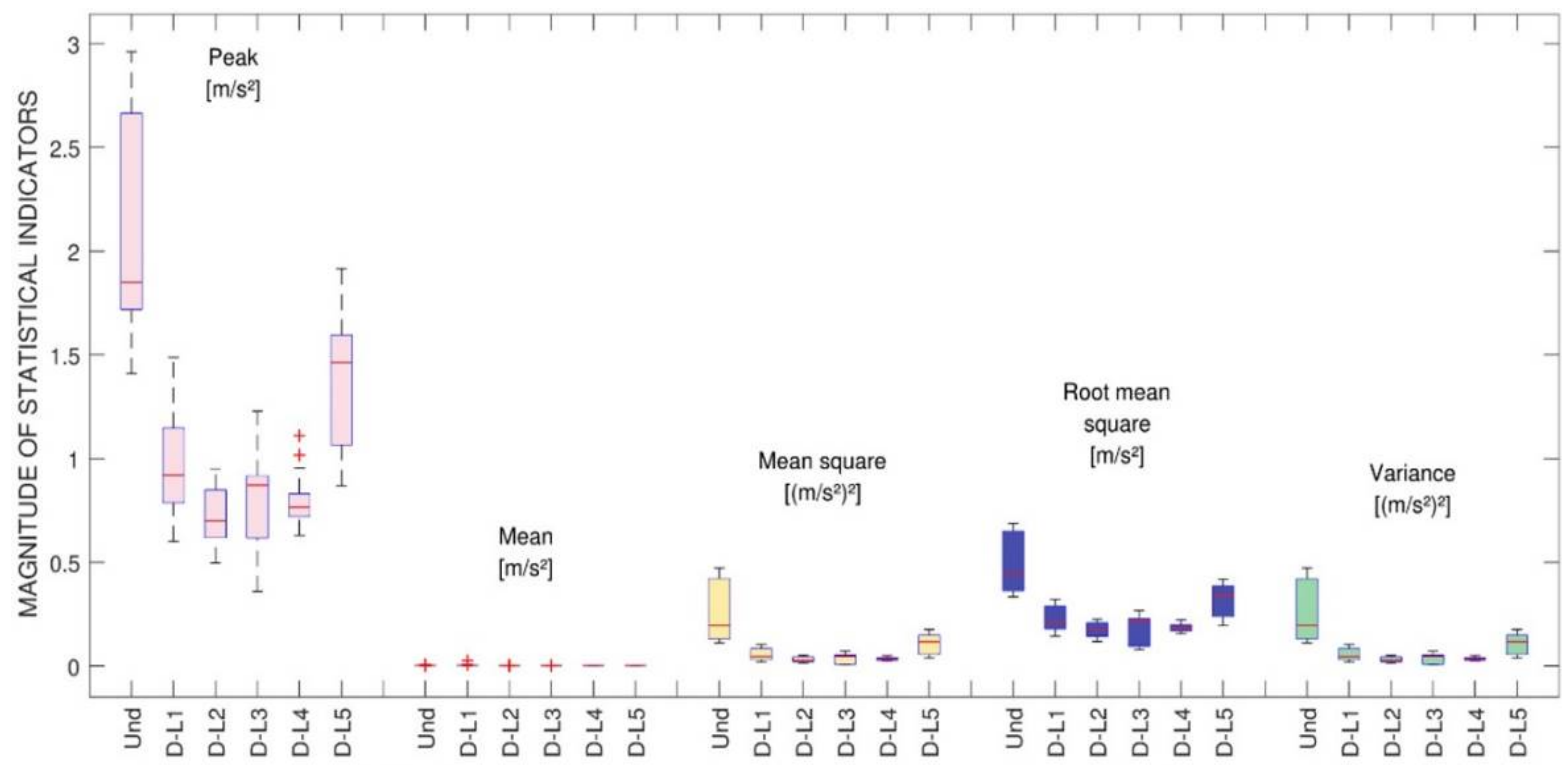

(a) Peak, mean, mean square, root mean square and variance.

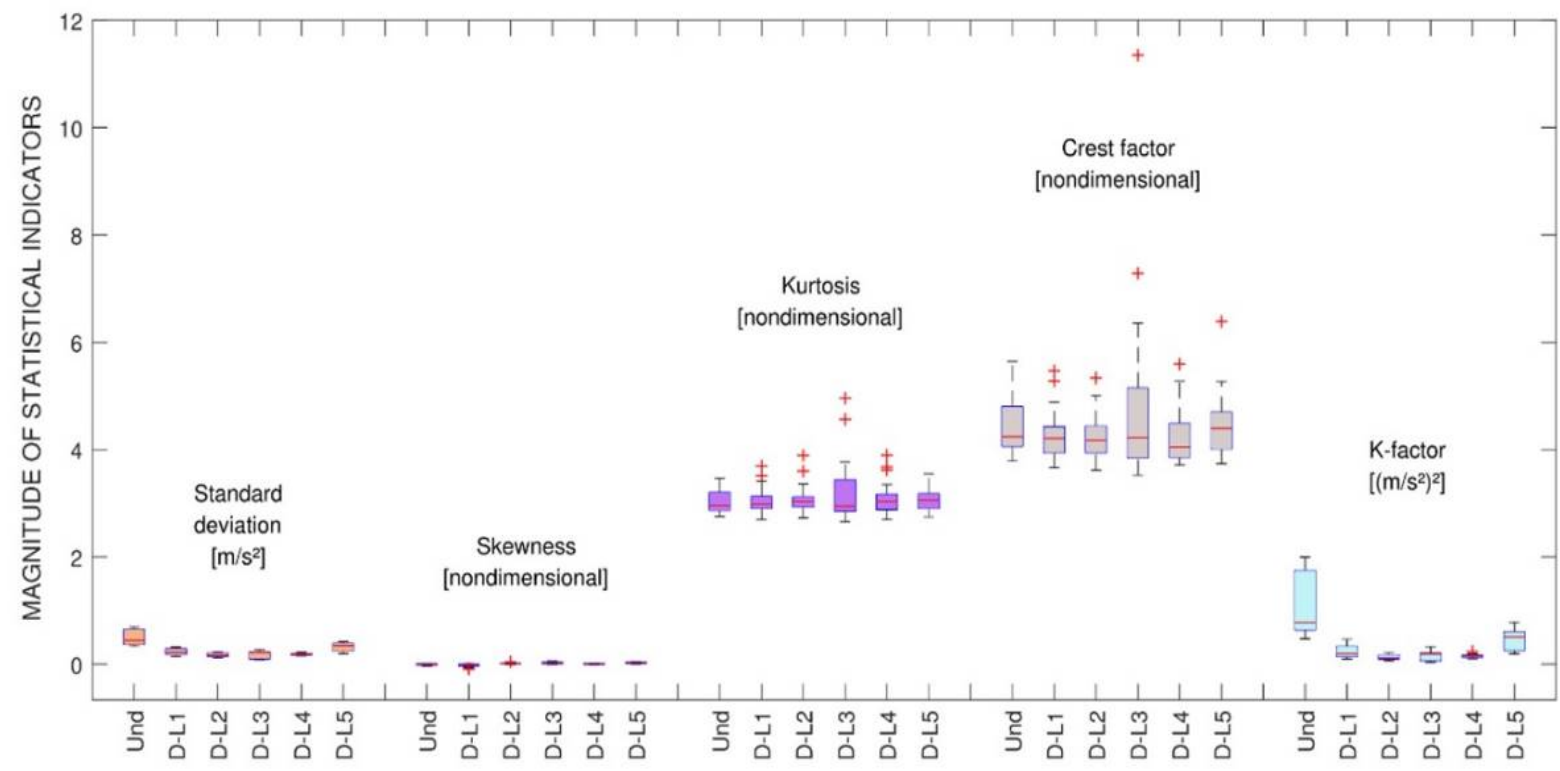

(b) Standard deviation, skewness, kurtosis, crest factor and k-factor.

Figure 11: Variation of the ten statistical indicators for signals from accelerometer AC3 of the experimental beam.

The implemented SVM was trained through Gaussian Radial Basis Function kernel using one-against-one strategy, also performing a 10-fold cross-validation. For this application, the best parameters of the SVM model are $\sigma=7.00$ and $\mathrm{C}=316.2278$. As explained in subsection "Numerical test: simply supported beam model", the SVM is an optimization technique that gives a unique and global solution, therefore the SVM performance is analyzed by interpreting the accuracy results, without any error convergence study.

\subsubsection{Results}

Table 3 contains the damage classification rates for the experimental steel beam problem. Both algorithms were executed 30 times in a 10-fold cross-validation and the classification rates are the mean values of these 30 repetitions. The mean values were calculated considering the number of correct classifications divided by the number of executions. The confusion matrices of the ANN and SVM models are presented in Fig. 13. They were assembled considering all test subsets of the cross-validation scheme, thus 5400 different examples were analyzed ( 18 examples for each test set $\times 10$ folds $\times 30$ models $=5400$ or 900 per damage scenario). 


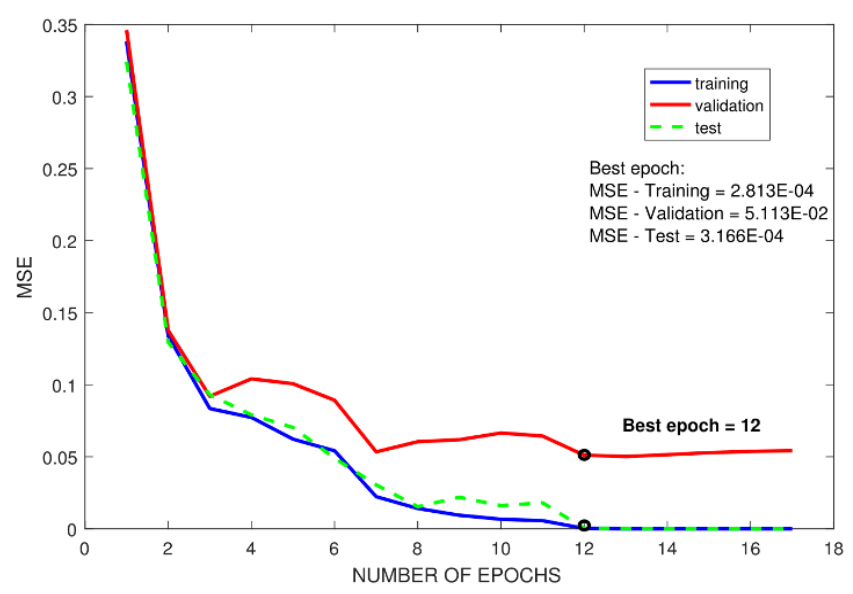

Figure 12: ANN performance progress for the experimental beam.

Table 3: Correct classification rate achieved for the experimental steel beam.

\begin{tabular}{ccccc}
\hline & Mean & $\begin{array}{c}\text { Standard } \\
\text { deviation }\end{array}$ & Max. & Min. \\
\hline ANN & $93.54 \%$ & $1.73 \%$ & $96.11 \%$ & $87.78 \%$ \\
SVM & $85.87 \%$ & $1.25 \%$ & $88.33 \%$ & $83.33 \%$ \\
\hline
\end{tabular}

\begin{tabular}{|c|c|c|c|c|c|c|c|}
\hline Und & $\begin{array}{c}862 \\
16.0 \%\end{array}$ & $\begin{array}{c}0 \\
0.0 \%\end{array}$ & $\begin{array}{c}1 \\
0.0 \%\end{array}$ & $\begin{array}{c}1 \\
0.0 \%\end{array}$ & $\begin{array}{c}0 \\
0.0 \%\end{array}$ & $\begin{array}{c}2 \\
0.0 \%\end{array}$ & $\begin{array}{c}99.5 \% \\
0.5 \%\end{array}$ \\
\hline \multirow{4}{*}{ 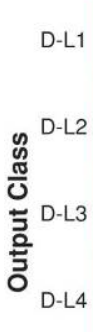 } & $\begin{array}{c}8 \\
0.1 \%\end{array}$ & $\begin{array}{c}864 \\
16.0 \%\end{array}$ & $\begin{array}{c}1 \\
0.0 \%\end{array}$ & $\begin{array}{c}4 \\
0.1 \%\end{array}$ & $\begin{array}{c}0 \\
0.0 \%\end{array}$ & $\begin{array}{c}2 \\
0.0 \%\end{array}$ & $\begin{array}{c}98.3 \% \\
1.7 \%\end{array}$ \\
\hline & $\begin{array}{c}6 \\
0.1 \%\end{array}$ & $\begin{array}{c}0 \\
0.0 \%\end{array}$ & $\begin{array}{c}782 \\
14.5 \%\end{array}$ & $\begin{array}{c}114 \\
2.1 \%\end{array}$ & $\begin{array}{c}1 \\
0.0 \%\end{array}$ & $\begin{array}{c}15 \\
0.3 \%\end{array}$ & $\begin{array}{l}85.2 \% \\
14.8 \%\end{array}$ \\
\hline & $\begin{array}{c}5 \\
0.1 \%\end{array}$ & $\begin{array}{c}29 \\
0.5 \%\end{array}$ & $\begin{array}{c}113 \\
2.1 \%\end{array}$ & $\begin{array}{c}778 \\
14.4 \%\end{array}$ & $\begin{array}{c}0 \\
0.0 \%\end{array}$ & $\begin{array}{c}12 \\
0.2 \%\end{array}$ & $\begin{array}{l}83.0 \% \\
17.0 \%\end{array}$ \\
\hline & $\begin{array}{c}3 \\
0.1 \%\end{array}$ & $\begin{array}{c}2 \\
0.0 \%\end{array}$ & $\begin{array}{c}1 \\
0.0 \%\end{array}$ & $\begin{array}{c}0 \\
0.0 \%\end{array}$ & $\begin{array}{c}899 \\
16.6 \%\end{array}$ & $\begin{array}{c}3 \\
0.1 \%\end{array}$ & $\begin{array}{c}99.0 \% \\
1.0 \%\end{array}$ \\
\hline \multirow[t]{3}{*}{ D-L5 } & $\begin{array}{c}16 \\
0.3 \%\end{array}$ & $\begin{array}{c}5 \\
0.1 \%\end{array}$ & $\begin{array}{c}2 \\
0.0 \%\end{array}$ & $\begin{array}{c}3 \\
0.1 \%\end{array}$ & $\begin{array}{c}0 \\
0.0 \%\end{array}$ & $\begin{array}{c}866 \\
16.0 \%\end{array}$ & $\begin{array}{l}97.1 \% \\
2.9 \%\end{array}$ \\
\hline & $\begin{array}{c}95.8 \% \\
4.2 \%\end{array}$ & $\begin{array}{c}96.0 \% \\
4.0 \%\end{array}$ & $\begin{array}{l}86.9 \% \\
13.1 \%\end{array}$ & $\begin{array}{l}86.4 \% \\
13.6 \%\end{array}$ & $\begin{array}{c}99.9 \% \\
0.1 \%\end{array}$ & $\begin{array}{c}96.2 \% \\
3.8 \%\end{array}$ & $\begin{array}{c}93.5 \% \\
6.5 \%\end{array}$ \\
\hline & Und & D-L1 & & $\begin{array}{l}\text { D-L3 } \\
\text { get Cl }\end{array}$ & $\begin{array}{l}\text { D-L4 } \\
\text { ss }\end{array}$ & D-L5 & \\
\hline
\end{tabular}

(a) ANN confusion matrix

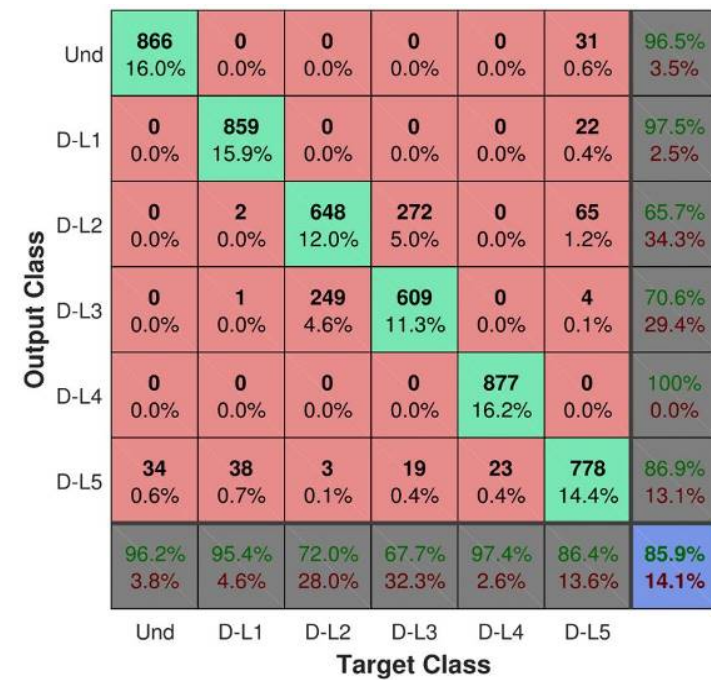

(b) SVM confusion matrix

Figure 13: Confusion matrices for the experimental steel beam.

According to the results, the ANN and SVM models were efficient in classifying the six different structural conditions. Despite the good percentages of correct classifications for both algorithms, the ANN had a quite superior performance when compared to SVM. The results increased from $85 \%$ (SVM) to $93 \%$ (ANN) with a small difference between standard deviations, from $1.25 \%$ (SVM) to $1.73 \%$ (ANN). By analyzing the confusion matrices for this example, one can observe that the accuracy percentage along the classes is slightly better in ANN than in SVM, on average, around 15.5\% against around $14.3 \%$, respectively, which shows the superiority of the ANN classifier for this application. In both algorithms, the worst performances are verified between "damaged signals - level 2" and "damaged signals - level 3", almost reaching $5 \%$ of all tested dataset for the ANN model and $10 \%$ for the SVM model. These results can be attributed to the fact that the damage imposed to the beam in the two scenarios were very similar (12 $\mathrm{mm}$ and $16 \mathrm{~mm}$ holes), making it difficult to classify these data correctly. 


\subsection{Experimental test II: railway bridge}

The studied structure in this section is an embedded steel bridge situated on the South-East high-speed track between the towns of Sens and Soucy, in France. A dynamic monitoring of this railway bridge was performed to analyze the influence of a strengthening procedure, as it can be seen in Fig. 14.

The strengthening procedure consisted in tightening special bearings in an effort to shift the first natural frequency from the excitation frequency due to train passing (Cury et al., 2010). Dynamic tests were conducted in the structure: 15 tests before strengthening and 13 tests after strengthening. The data comprised the measurements of eight vertical accelerometers and two horizontal accelerometers (longitudinal and transversal) located under the bridge deck, acquired at a sampling rate of $4096 \mathrm{~Hz}$, respecting the Nyquist frequency. Since the experimental tests were recorded during the passage of a high-speed train, the ambient vibrations are not controlled (output-only accelerations). Therefore, repeatability tests as well as coherence analyses were not evaluated. A typical response of the structure is illustrated in Fig. 15.

a)

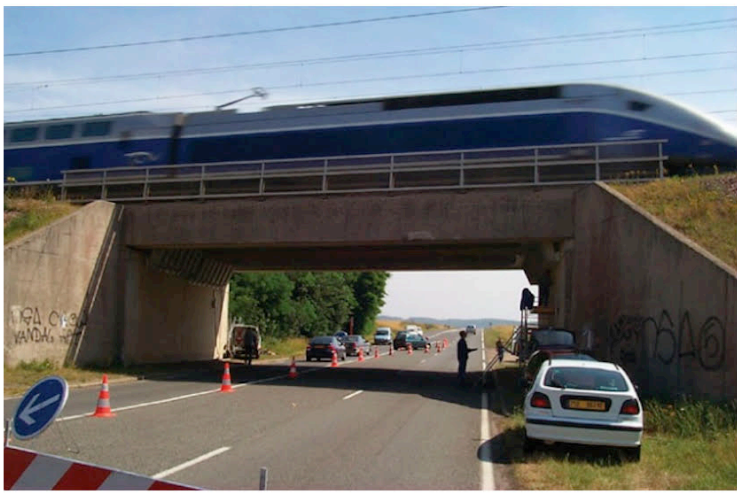

b)

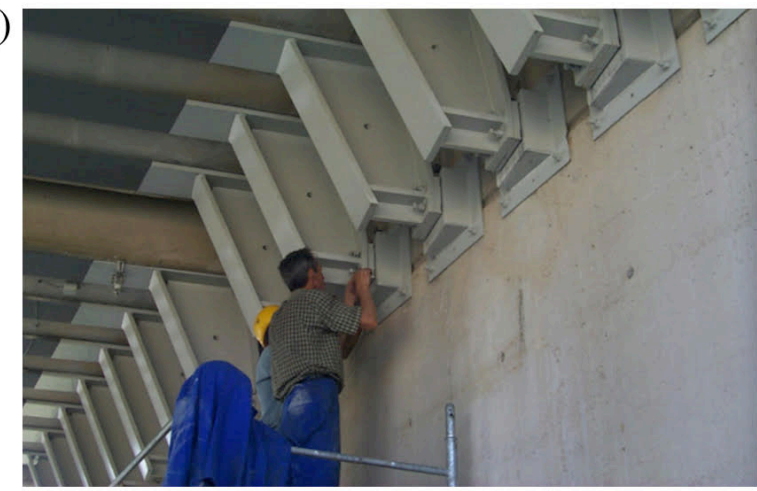

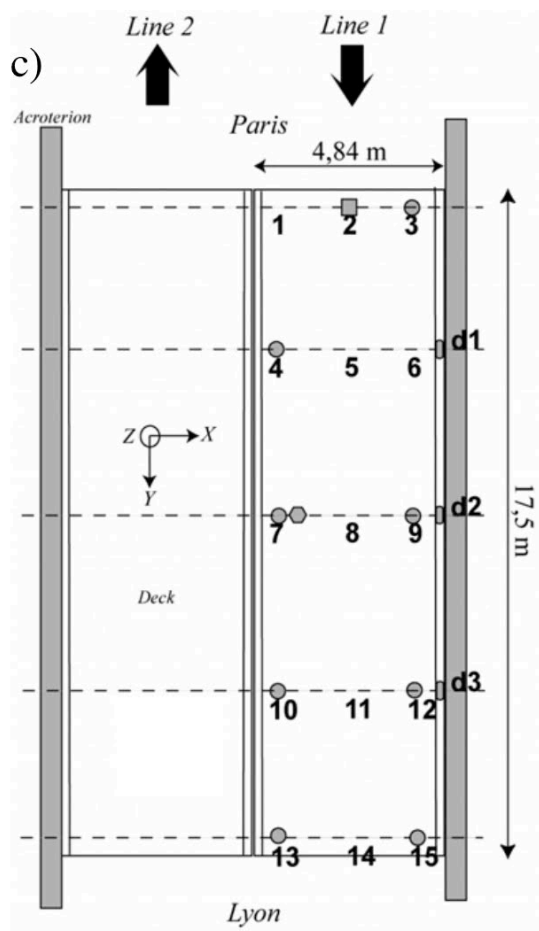

Vertical acceleration transducer ( $\mathrm{Z}$ axis)

- Transversal acceleration transducer ( $\mathrm{X}$ axis)

$\square$ Longitudinal acceleration transducer ( $\mathrm{Y}$ axis)

Figure 14: Experimental setup of the bridge (Cury et al., 2010). (a) View of the bridge during the strengthening procedure; (b) Strengthening system; (c) Localization of the sensors.

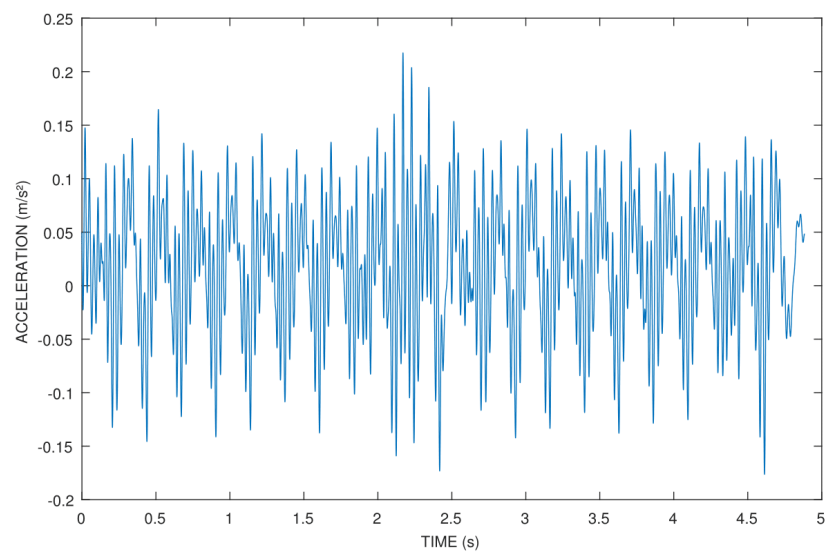

Figure 15: Typical response of the railway bridge. 
Taking into account that the algorithms need a reasonable amount of samples and the collected signals of the bridge have less than one minute, the time histories of each accelerometer are separately considered. In this case, the methodology of cutting the signals in order to increase the quantity of data was not applied once the short-registered time histories do not allow this strategy. Thus, the ten statistical indicators were evaluated for each accelerometer signal, yielding an input matrix [280×11] as demonstrated in Eq. (6), where the rows are the samples (the dynamic tests), the first ten columns are the statistical indicators and the last column indicates the position associated with accelerometer where the respective response was measured (from 1 to 10). Fig. 16 shows the variation of the statistical parameters between the two structural conditions. The targets for the bridge were encoded as: 0 - before strengthening and 1 - after strengthening.

$$
\left[\begin{array}{cccc}
T_{1} S I_{1} & T_{1} S I_{2} & \cdots & T_{1} S I_{11} \\
T_{2} S I_{1} & T_{2} S I_{2} & \cdots & T_{2} S I_{11} \\
\vdots & \vdots & \ddots & \vdots \\
T_{280} S I_{1} & T_{280} S I_{2} & \cdots & T_{280} S I_{11}
\end{array}\right]
$$

The ANN and SVM for the bridge have the same architecture and parameters of those applied in the beam examples, except for the number of neurons and the RBF parameters. In this case, the MLP has 15 neurons in the hidden layer and the SVM has $\sigma=2.00$ and $C=316.2278$. Fig. 17 exhibits a convergence curve based on the model approximation errors in the training, validation and testing stages for the ANN model. As it can be observed, the training stops when the validation error starts to increase (at epoch 8), preventing a possible data overfitting.

\subsubsection{Results}

Table 4 and Fig. 18 show the accuracy values and the confusion matrices obtained by SVM and ANN. Just as the other applications, the algorithms were executed 30 times with 10-fold cross-validation and the table results are the values of correct classifications divided by the number of executions. The confusion matrices are constructed considering all test subsets of the cross-validation scheme, resulting in 4500 analyzed examples before strengthening (15 for each test set $\times 10$ folds $\times 30$ models) and in 3900 after strengthening (13 for each test set $\times 10$ folds $\times 30$ models).

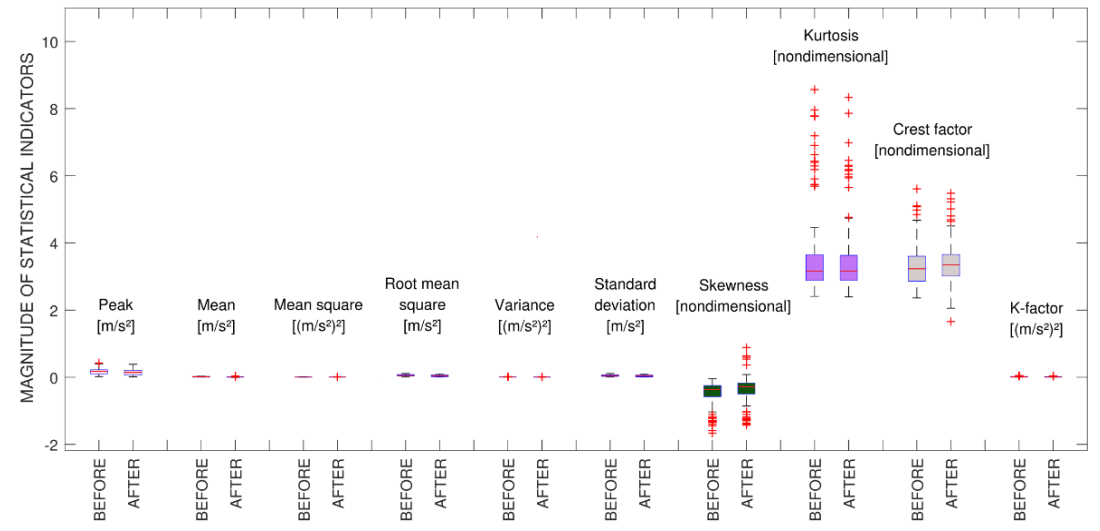

Figure 16: Variation of the ten statistical indicators for signals from the railway bridge. 


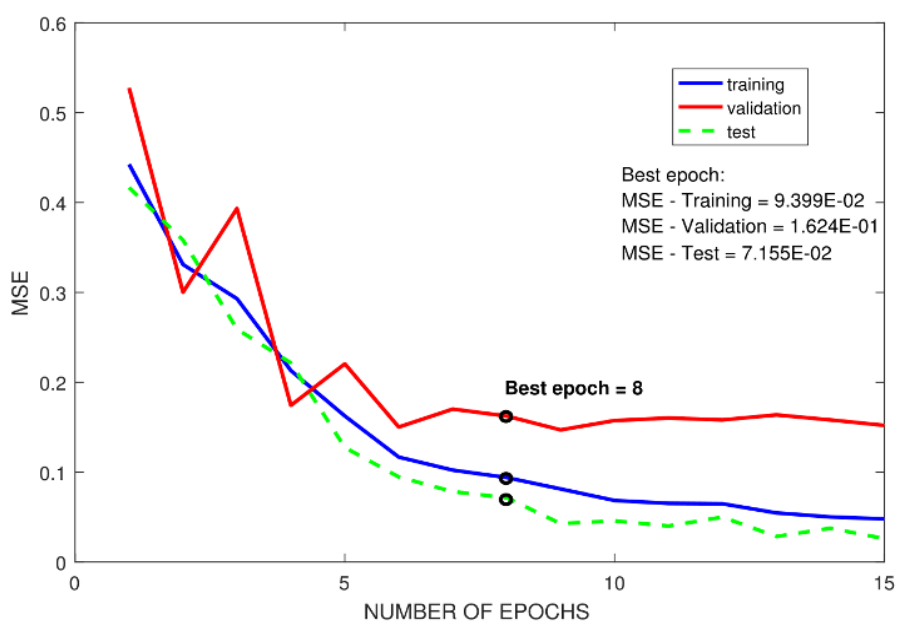

Figure 17: ANN performance progress for the railway bridge.

Table 4: Correct classification rate achieved for the experimental tested railway bridge.

\begin{tabular}{ccccc}
\hline & Mean & $\begin{array}{c}\text { Standard } \\
\text { deviation }\end{array}$ & Max. & Min. \\
\hline ANN & $87.09 \%$ & $1.35 \%$ & $90.36 \%$ & $85.00 \%$ \\
SVM & $80.24 \%$ & $1.29 \%$ & $82.86 \%$ & $77.86 \%$ \\
\hline
\end{tabular}

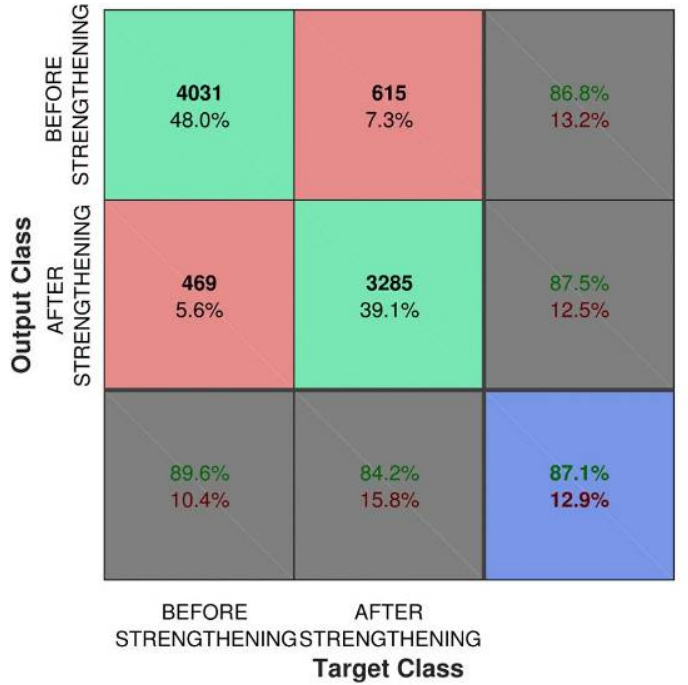

(a) ANN confusion matrix

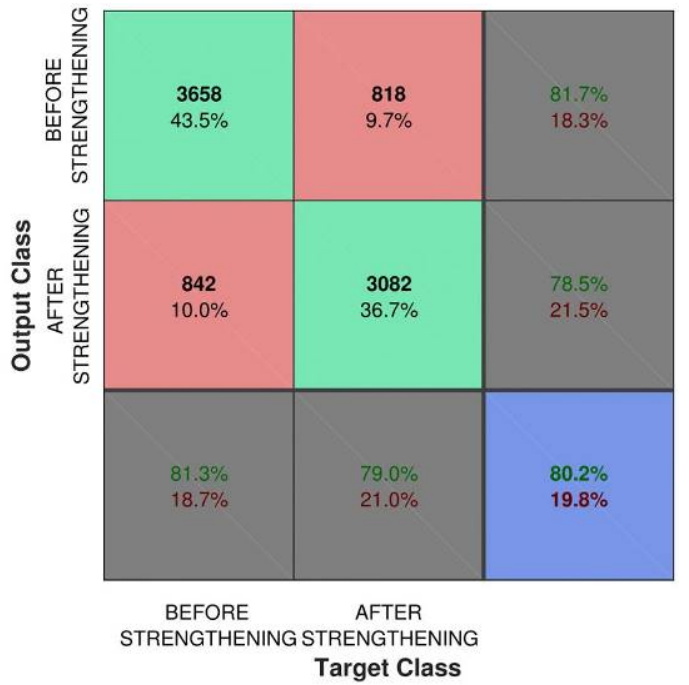

(b) SVM confusion matrix

Figure 18: Confusion matrices for the railway bridge.

Both methods had similar performances, around $80 \%$. However, the ANN results are slightly better than those of the SVM, increased from $80.24 \%$ (SVM) to $87.09 \%$ (ANN). A comparison between the two structural configurations can be made by interpreting the confusion matrices, in which the accuracy percentage for each class is similar for both algorithms, around $40 \%$. For the SVM model, the worst performance is when 842 signals were classified as being "before strengthening" instead "after strengthening" (false-negative), representing $10 \%$ of all tested data. Meanwhile, for the ANN model, the worst performance is when 615 signals were classified as being "after strengthening" instead "before strengthening" (false-positive), representing $7.3 \%$ of all tested dataset. 


\section{CONCLUSIONS}

In this paper, a strategy to detect structural changes based on statistical indicators and machine learning methods was investigated. Ten statistical parameters extracted from structural raw acceleration measurements were used as inputs to the two classification methods: Artificial Neural Network (ANN) and Support Vector Machine (SVM). The proposed approach was evaluated by comparing the results through three applications: a numerical model of a simply supported beam with different levels of damage, an experimental investigation of a steel beam carried out in laboratory and an experimental tested railway bridge submitted to a strengthening procedure.

In general, both methods obtained good results and had similar performances for all structural change identification problems, achieving classification rates greater than $80 \%$. By analyzing the results, SVM had better performance than ANN only for the numerical beam model. However, for the second and third structures (steel beam and railway bridge) ANN showed a better performance. This type of results is essentially inherent to the classification method and to the problem under analysis. Thus, there is no general rule. For the numerical example, the SVM's separation hyperplane (and margins) were more easily adjusted for the numerical data, which did not occur for the experimental cases.

In practice, the main advantage of the proposed approach is to deal with dynamic data without needing to perform a modal identification procedure. Although the statistical parameters do not present significant visual variation in some cases, they are able to represent subtle differences among the vibration signals, allowing the machine learning methods to detect structural alterations caused by the occurrence of damage or a strengthening procedure. The good average classification performance reflects the effectiveness of the presented strategy and motivates the development of computational tools using statistical analysis for SHM. Notwithstanding the good results, this approach has some limitations such as the large amount of data to training the models and, as in all methods based on supervised learning, the need of prior knowledge of the structural behaviors (targets). Thus, a further study of these aspects should be considered in future works.

\section{ACKNOWLEDGEMENTS}

This study was financed in part by the Coordenação de Aperfeiçoamento de Pessoal de Nível Superior - Brasil (CAPES) - Finance Code "Edital Procad 071/2013", by the Conselho Nacional de Desenvolvimento Científico e Tecnológico - "National Council of Technological and Scientific Development" (CNPq), by the Fundação de Amparo à Pesquisa do Estado de Minas Gerais (FAPEMIG) - Funding "PPM-0002-16" and "PPM-00106-17" - and by the Universidade Federal de Juiz de Fora - Programa de Pós-Graduação em Modelagem Computacional (UFJF-PGMC). Moreover, the authors would like to thank to COPPE-UFRJ (Universidade Federal do Rio de Janeiro) and to IFSTTAR (former LCPC - Laboratoire Central des Ponts et Chaussées) and to SNCF (Société Nationale des Chemins de fer Français) - project 01V0527 RGCU “Evaluation dynamique des ponts" - for the data used in this paper.

\section{REFERENCES}

Allemang, R. J. (2003). The modal assurance criterion - twenty years of use and abuse. Sound and Vibration, 37(8), 14-23.

Alves, V., Cury, A., Roitman, N., Magluta, C., Cremona, C. (2015). Structural modification assessment using supervised learning methods applied to vibration data. Engineering Structures, 99, 439-448.

Amezquita-Sanchez, J. P., Adeli, H. (2016). Signal processing techniques for vibration-based health monitoring of smart structures. Archives of Computational Methods in Engineering, 23(1), 1-15.

Battista, R. C., Pfeil, M. S. (2002). Monitoring and control of wind-induced oscillations of a steel bridge. In Proceedings of the fourth European Conference on Structural Dynamics-EURODYN'2002, 925-930.

Bishop, C. M. (2006). Pattern recognition and machine learning. Springer-Verlag.

Cabboi, A., Magalhães, F., Gentile, C., Cunha, Á. (2017). Automated modal identification and tracking: Application to an iron arch bridge. Structural Control and Health Monitoring, 24(1), e1854.

Cachot, E., Vayssade, T., Virlogeux, M., Lancon, H., Hajar, Z., Servant, C. (2015). The Millau viaduct: ten years of structural monitoring. Structural Engineering International, 25(4), 375-380.

Cardoso, R., Cury, A., Barbosa, F. (2017). A robust methodology for modal parameters estimation applied to SHM. Mechanical Systems and Signal Processing, 95, 24-41. 
Cury, A. A., Borges, C. C., Barbosa, F. S. (2011). A two-step technique for damage assessment using numerical and experimental vibration data. Structural Health Monitoring, 10(4), 417-428.

Cury, A., Crémona, C., Diday, E. (2010). Application of symbolic data analysis for structural modification assessment. Engineering Structures, 32(3), 762-775.

Doebling, S. W., Farrar, C.R., Prime, M. B., Shevitz, D. (1998). A review of damage identification methods that examine changes in dynamic properties. Shock and Vibration Digest, 30(2), 91-105.

Farrar, C. R., Worden, K. (2012). Structural health monitoring: a machine learning perspective. John Wiley \& Sons.

Hagan, M. T., Menhaj, M. B. (1994). Training feedforward networks with the Marquardt algorithm. IEEE transactions on Neural Networks, 5(6), 989-993.

Kohavi, R. (1995). A study of cross-validation and bootstrap for accuracy estimation and model selection. In Proceedings of the 14th International Joint Conference on Artificial Intelligence - IJCAI, 14(2), 1137-1145.

Ni, Y. Q., Xia, H. W., Wong, K. Y., Ko, J. M. (2011). In-service condition assessment of bridge deck using long-term monitoring data of strain response. Journal of Bridge Engineering, 17(6), 876-885.

Pandey, A. K., Biswas, M. (1994). Damage detection in structures using changes in flexibility. Journal of Sound and Vibration, 169(1), 3-17.

Peeters, B., De Roeck, G. (1999). Reference-based stochastic subspace identification for output-only modal analysis. Mechanical Systems and Signal Processing, 13(6), 855-878.

Principe, J. C., Euliano, N. R., Lefebvre, W. C. (2000). Neural and adaptive systems: fundamentals through simulations. John Wiley \& Sons.

Rytter, A. (1993). Vibrational based inspection of civil engineering structures, Ph.D. Thesis, Aalborg University, Denmark.

Santos, A., Figueiredo, E., Silva, M. F. M., Sales, C. S., Costa, J. C. W. A. (2016). Machine learning algorithms for damage detection: Kernel-based approaches. Journal of Sound and Vibration, 363, 584-599.

Vapnik, V. (1995). The nature of statistical learning theory. Springer-Verlag.

Worden, K., Lane, A. J. (2001). Damage identification using support vector machines. Smart Materials and Structures, 10(3), 540 .

Zang, C., Friswell, M. I., Imregun, M. (2004). Structural damage detection using independent component analysis. Structural Health Monitoring, 3(1), 69-83. 